\title{
EVALUATION OF NONEQUILIBRIUM BOUNDARY CONDITIONS FOR HYPERSONIC RAREFIED GAS FLOWS
}

\author{
N. T. P. Le, Ch. J. Greenshields, and J. M. Reese \\ Department of Mechanical Engineering \\ University of Strathclyde \\ Glasgow G1 1XJ, United Kingdom
}

\begin{abstract}
A new Computational Fluid Dynamics (CFD) solver for high-speed viscous flows in the OpenFOAM code is validated against published experimental data and Direct Simulation Monte Carlo (DSMC) results. The laminar flat plate and circular cylinder cases are studied for Mach numbers, Ma, ranging from 6 to 12.7, and with argon and nitrogen as working gases. Simulation results for the laminar flat plate cases show that the combination of accommodation coefficient values $\sigma_{u}=0.7$ and $\sigma_{T}=1.0$ in the Maxwell/Smoluchowski conditions, and the coefficient values $A_{1}=1.5$ and $A_{2}=1.0$ in the second-order velocity slip condition, give best agreement with experimental data of surface pressure. The values $\sigma_{u}=0.7$ and $\sigma_{T}=1.0$ also give good agreement with DSMC data of surface pressure at the stagnation point in the circular cylinder case at $\mathrm{Kn}=0.25$. The Langmuir surface adsorption condition is also tested for the laminar flat plate case, but initial results were not as good as the Maxwell/Smoluchowski boundary conditions.
\end{abstract}

\section{INTRODUCTION}

The efficient and accurate simulation of hypersonic rarefied gas flow is important in the aerodynamic design of space vehicles; in particular, predictions of temperature and pressure experienced by the surface of the vehicle. There are different techniques for simulating these flows, including the DSMC method and CFD. Most successful is the DSMC method; however, the computational time required is much longer than for CFD, which generally solves the Navier-Stokes-Fourier (N-S-F) hydrodynamic equations. The translational thermodynamic nonequilibrium of the gas flow can be characterized by the Knudsen number:

$$
\mathrm{Kn}=\frac{\lambda}{L} \approx \frac{\lambda}{Q}|\nabla Q|
$$

This is an Open Access article distributed under the terms of the Creative Commons Attribution-Noncommercial License 3.0, which permits unrestricted use, distribution, and reproduction in any noncommercial medium, provided the original work is properly cited. 
where $\lambda$ is the mean free path; $L$ is the macroscopic characteristic length scale; and $Q$ is the quantity of interest such as the gas temperature, surface, and density.

At low altitudes, the gas density is relatively high, $\mathrm{Kn}$ is small, and gas flows may be effectively simulated by solving the Euler equations (when $\mathrm{Kn} \leq 0.001$ ) or the N-S-F equations with no-slip boundary conditions (when $0.001 \leq \mathrm{Kn}$ $\leq 0.01$ ). At high altitude, the gas density is lower, the mean free path, $\lambda$, is large, and nonequilibrium behavior increases. An approach to improve $\mathrm{N}-\mathrm{S}-\mathrm{F}$ predictions in the range $(0.01 \leq \mathrm{Kn} \leq 0.1)$ is to apply velocity slip and temperature jump boundary conditions at aerodynamic surfaces.

Nonequilibrium velocity slip and temperature jump boundary conditions include those proposed by Maxwell [1], Smoluchowski [2], Myong [3], and "second-order" velocity slip conditions [4]. The problem with the Maxwell/ Smoluchowski (M/S) boundary conditions is that the free parameters, the tangential momentum, $\sigma_{u}$, and thermal, $\sigma_{T}$, accommodation coefficients, critically affect simulation results. A similar problem with the second-order velocity slip boundary condition is the values of the coefficients of the first and second order terms. The Langmuir boundary condition due to Myong [3] overcomes this problem as the velocity slip and temperature jump coefficients are determined through a Langmuir adsorption isotherm model that gives good results for certain rarefied gas microflows.

In this paper, the two-dimensional (2D) numerical investigations of the velocity slip and temperature jump boundary conditions are presented in conjunction with the $\mathrm{N}-\mathrm{S}-\mathrm{F}$ equations to propose the best coefficients for hypersonic rarefied gas flow. The test cases are a circular cylinder in cross-flow at $\mathrm{Kn}=0.25$ and the laminar sharp-leading-edge flat plate. In the flat plate case, the local Knudsen number (i. e., with $L$ in Eq. (1) a running distance from the leading edge) is much larger than the overall Knudsen number (i. e., with the characteristic length being the total streamwise length of the flat plate). For these sharp-leading-edge geometries, the authors' CFD predictions are expected to agree reasonably with the experimental data due to the increased nonequilibrium behavior near the leading edge. The simulation results will be compared with DSMC [5] and experimental data for the flat plate case $[6,7]$. The flow conditions of the experiments $[6,7]$, such as freestream temperature, $T_{\infty}$, freestream pressure, $p_{\infty}$, and freestream mean free path, $\lambda_{\infty}$, are shown in Table 1 .

Table 1 Flow conditions in previous flat-plate experiments

\begin{tabular}{crccccc}
\hline Researchers & $\mathrm{Ma}$ & $T_{\infty}, \mathrm{K}$ & $p_{\infty}, \mathrm{Pa}$ & $\lambda_{\infty}, \mathrm{mm}$ & $T_{w}, \mathrm{~K}$ & Gas \\
\hline Becker [6] & 12.7 & 64.5 & 3.73 & 0.23 & 292 & Argon \\
Metcalf et al. $[7]$ & 6.1 & 83.4 & 2.97 & 0.35 & 77 & Nitrogen \\
\hline
\end{tabular}




\section{REVIEW OF NONEQUILIBRIUM BOUNDARY CONDITIONS}

Most nonequilibrium slip/jump boundary conditions can be expressed in the form:

$$
\phi+a \nabla_{\mathbf{n}}(\mathbf{S} \cdot \phi)=\Phi
$$

where $\phi$ is the variable of interest, e.g., velocity or temperature; $\nabla \mathbf{n} \equiv \mathbf{n} \cdot \nabla$ is the component of the gradient normal to boundary surface with $\mathbf{n}$ being the unit normal vector defined as positive in the direction pointing out of the flow domain; $a$ is the coefficient specific to each boundary condition; and $\Phi$ is the limiting value in the case of no-slip/jump, e.g., the wall velocity or temperature. Tensor $\mathbf{S}=\mathbf{I}-\mathbf{n n}$ where $\mathbf{I}$ is the identity tensor, removes normal components of any nonscalar field, e.g., velocity, so that slip only occurs in the direction tangential to the surface. The normal gradient can be expressed numerically as:

$$
\nabla_{\mathbf{n}} \phi=C_{\Delta}\left(\phi-\phi_{i}\right)
$$

where the subscript ' $i$ ' denotes a value in the numerical cell adjacent to the boundary face of the solid surface, and $C_{\Delta}=1 /|\mathbf{d}|$, with $\mathbf{d}$ the distance from the numerical cell center to the boundary face center of the solid surface.

Experimental observations show that the gas temperature at the surface is not equal to the surface temperature. This difference is called the "temperature jump" and is driven by the heat flux normal to the surface. The Smoluchowski model [2] for the temperature jump can be written:

$$
T+\frac{2-\sigma_{T}}{\sigma_{T}} \frac{2 \gamma}{(\gamma+1) \operatorname{Pr}} \lambda \nabla_{\mathbf{n}} T=T_{w}
$$

where $\sigma_{T}$ is the thermal accommodation coefficient $0 \leq \sigma_{T} \leq 1.0 ; T$ is the gas temperature at the surface; $T_{w}$ is the surface temperature; $\operatorname{Pr}$ is the Prandtl number; $\gamma$ is the specific heat ratio; and $\lambda$ is the mean free path at the wall. There are a number of different definitions of the mean free path $\lambda$; here, the Maxwellian is used:

$$
\lambda=\frac{\mu}{\rho} \sqrt{\frac{\pi}{2 R T}}
$$

where $\rho$ is the gas density at the surface; $R$ is the specific gas constant; and $\mu$ is the gas viscosity at the surface.

The Maxwell slip boundary condition [1], including the effect of thermal creep, can be written in vector form as:

$$
\mathbf{u}=-\frac{2-\sigma_{u}}{\sigma_{u}} \frac{\lambda \boldsymbol{\tau}}{\mu}-\frac{3}{4} \frac{\operatorname{Pr}(\gamma-1)}{\gamma p} \mathbf{q}+\mathbf{u}_{w}
$$


where $\mathbf{u}$ is the velocity; the tangential shear stress is $\boldsymbol{\tau}=\mathbf{S} \cdot(\mathbf{n} \cdot \mathbf{\Pi})$ and the heat flux is $\mathbf{q}=\mathbf{Q} \cdot \mathbf{S}$ at the surface; with bold type face denoting a vector quantity; $\boldsymbol{\Pi}$ is the stress tensor at the surface; $\mathbf{Q}$ is the heat flux vector along the surface; $p$ is the gas pressure at the surface; and $\mathbf{u}_{w}$ is the surface velocity. The tangential momentum accommodation coefficient, $\sigma_{u}$, determines the proportion of molecules reflected from the surface specularly $\left(1-\sigma_{u}\right)$ or diffusely $\left(\sigma_{u}\right)$, and $0 \leq \sigma_{u} \leq 1$

Equation (4) can be expressed in the form of Eq. (2) by substituting $\tau=$ $\mathbf{S} \cdot(\mathbf{n} \cdot \boldsymbol{\Pi})$ and $\boldsymbol{\Pi}=\mu \nabla \mathbf{u}+\boldsymbol{\Pi}_{m c}$, with $\boldsymbol{\Pi}_{m c}=\mu(\nabla \mathbf{u})^{\mathrm{T}}-(2 / 3) \mathbf{I t r}(\nabla \mathbf{u})$ into Eq. (4) where the superscript $\mathrm{T}$ denotes the transpose and tr denotes the trace. Noting that $\mathbf{S} \cdot \nabla_{\mathbf{n}} \phi \equiv \nabla_{\mathbf{n}}(\mathbf{S} \cdot \phi)$, Eq. (4) becomes:

$$
\mathbf{u}+\frac{2-\sigma_{u}}{\sigma_{u}} \lambda \nabla_{\mathbf{n}}(\mathbf{S} \cdot \mathbf{u})=\mathbf{u}_{w}-\frac{2-\sigma_{u}}{\sigma_{u}} \frac{\lambda}{\mu} \mathbf{S} \cdot\left(\mathbf{n} \cdot \boldsymbol{\Pi}_{m c}\right)-\frac{3}{4} \frac{\mu}{\rho} \frac{\mathbf{S} \cdot \nabla T}{T} .
$$

The right-hand side contains three terms that are associated with (in order): the wall velocity, the so-called curvature effect [8], and the thermal creep.

Myong's boundary conditions for velocity slip and temperature jump are based on the Langmuir adsorption isotherm model. Adsorption is the process by which free gas molecules attach to a solid surface, and the boundary conditions are expressed as [3]:

$$
\phi=(1-\alpha) \phi_{g}+\alpha \phi_{w}
$$

where the subscript ' $g$ ' denotes a reference value in the gas; $\alpha$ is the fraction of coverage (i. e., occupied surface sites, $0 \leq \alpha \leq 1$ ) calculated by [3] for monatomic gas:

$$
\alpha=\frac{\beta p}{1+\beta p} .
$$

Here, $\beta$ is the equilibrium constant relating to the surface temperature and (measured) heat of adsorption $D_{e}(\mathrm{~J} / \mathrm{mol})$ and is calculated as follows:

$$
\beta=\frac{A \lambda}{R_{u} T_{w}} \exp \left(\frac{D_{e}}{R_{u} T_{w}}\right)
$$

where $R_{u}$ is the universal gas constant $(\mathrm{J} /(\mathrm{kmol} \cdot \mathrm{K})) ; A$ is the mean area of a site $\left(\mathrm{m}^{2} / \mathrm{mol}\right)$ and is either measured or calculated approximately by $N_{A} \pi d^{2} / 4[3]$ for monatomic and diatomic gases, with $d$ the molecular diameter and $N_{A}$ the Avogadro number.

If $\phi_{g}$ is taken as a reference value of a mean free pathaway in the direction normal to the surface, then $\phi-\phi_{g}=\lambda \nabla_{\mathbf{n}} \phi$. Equation (6) can, therefore, be expressed by:

$$
\phi+\frac{\lambda}{\beta p} \nabla_{\mathbf{n}} \phi=\phi_{w} .
$$


Table 2 Coefficient $a$ in Eq. (2) for various slip velocity and temperature jump boundary conditions

\begin{tabular}{ccc}
\hline Velocity slip & Temperature jump & Coefficient $a$ \\
\hline- & Smoluchowski & $\lambda \frac{2-\sigma_{T}}{\sigma_{T}} \frac{2 \gamma}{(\gamma+1) \operatorname{Pr}}$ \\
Maxwell & - & $\frac{\lambda\left(2-\sigma_{u}\right)}{\sigma_{u}}$ \\
Langmuir & Langmuir & $\frac{\lambda}{\beta p}$ \\
Second-order & - & $A_{1} \lambda+A_{2} \lambda^{2} C_{\Delta}$ \\
\hline
\end{tabular}

So, the Langmuir boundary condition has the same form as the Maxwell boundary condition without the thermal creep and the curvature effect indicated by the term $\boldsymbol{\Pi}_{m c}$ in Eq. (5), i. e., the same form as the general Eq. (2), except for $\left(2-\sigma_{u}\right) / \sigma_{u}$ replaced by $1 /(\beta p)$. The coefficient for the Langmuir boundary condition is calculated from Eq. (7) which depends on the values of $A$ and $D_{e}$, whereas $\sigma_{u}$ is the only free parameter in the Maxwell boundary condition.

It is seen that all first-order velocity slip and temperature jump boundary conditions have the same general form as in Eq. (2), but with different coefficients $a$.

The second-order velocity slip boundary condition for planar surfaces is [4]:

$$
\mathbf{u}+A_{1} \lambda \nabla_{\mathbf{n}}(\mathbf{S} \cdot \mathbf{u})+A_{2} \lambda^{2} \nabla_{\mathbf{n}}^{2}(\mathbf{S} \cdot \mathbf{u})=\mathbf{u}_{w}
$$

where $A_{1}$ and $A_{2}$ are the slip coefficients for the first- and second-order terms, respectively. Their values are proposed either from theory or from experiment and are still the subject of much discussion. Equation (9) is expressed in the general form of Eq. (2) by substituting $\nabla_{\mathbf{n}} \mathbf{u}=C_{\Delta}\left(\mathbf{u}-\mathbf{u}_{i}\right)$ into Eq. (9):

$$
\mathbf{u}+\left(A_{1} \lambda+A_{2} \lambda^{2} C_{\triangle}\right) \nabla_{\mathbf{n}}(\mathbf{S} \cdot \mathbf{u})=\mathbf{u}_{w}+A_{2} \lambda^{2} C_{\Delta} \nabla_{\mathbf{n}}(\mathbf{S} \cdot \mathbf{u})_{i} .
$$

From Eqs. (3), (5), (8), and (10), the specific coefficients $a$ for the different models for velocity slip and temperature jump boundary conditions in general Eq. (2) are shown in Table 2.

\section{NUMERICAL IMPLEMENTATION AND SIMULATION RESULTS}

In this work, OpenFOAM is used, the open source CFD software [9], with finite volume (FV) numerics to solve systems of partial differential equations ascribed 
on any three-dimensional unstructured mesh of polygonal cells. The FV discretization is based on Gaussian integration and, so, uses values and normal gradients of fields at cell faces. If the face belongs to a boundary, the face value and gradient required by the discretization procedure must be obtained from the boundary condition. Various fundamental types of boundary conditions are implemented in OpenFOAM, including one known as "partial slip," that is a mixture of a fixed value, or Dirichlet, condition and a zero-gradient condition a Neumann condition where the normal gradient is zero. The "mixing" is controlled by a fraction coefficient $\theta(0 \leq \theta \leq 1)$ where $\theta=0$ for the zero-gradient condition and $\theta=1$ for fixed value condition. A reference value is also required that is assigned to the fraction of the boundary condition that is a fixed value.

For the case of scalar fields, this boundary condition can be used as the basis for any slip/jump boundary condition described by Eq. (2), by setting

$$
\theta=1-\frac{a C_{\Delta}}{1+a C_{\Delta}}
$$

and setting the value $\Phi$ to be the reference value for the fixed value component. For the case of vector fields, the standard partial slip condition was modified to include the tensor $\mathbf{S}$, so that only the tangential components of the field are "slipped." In either case, the fraction values, $\theta$, for the boundary conditions considered are simply determined by Eq. (11) with the values of $a$ from Table 2. The $\mathrm{N}-\mathrm{S}-\mathrm{F}$ equations are implemented and solved numerically in OpenFOAM using a FV discretization and high-resolution central schemes that are described in detail in [10]. These are implemented as the solver rhoCentralFoam in OpenFOAM for simulating high-speed viscous flows. A calorically perfect gas is considered for all simulations in the present study; so, $p=\rho R T$ and $e=c_{v} T=(\gamma-1) R T$. The Sutherland law is used for modeling the dynamic viscosity in the flat plate case, i. e.,

$$
\mu=A_{S} \frac{T^{1.5}}{T+T_{S}}
$$

where $A_{S}$ and $T_{S}$ are the constants. The coefficient of thermal conductivity is then computed from the standard equation:

$$
k=\frac{c_{p} \mu}{\operatorname{Pr}} .
$$

The values $A_{S}, T_{S}, R, \gamma$, and $\operatorname{Pr}$ of the working gases in the flat-plate cases are given in Table $3[8,11,12]$.

The power law is used for modeling the viscosity in the cylinder case, in order to compare with DSMC in [5], i. e.,

$$
\mu=A_{P} T^{s}
$$

where $s=0.734$ and $A_{P}=0.32 \cdot 10^{-6}\left(\mathrm{~Pa} \cdot \mathrm{s} / \mathrm{K}^{s}\right)$ are calculated from [5]. 
Table 3 Coefficients for gas transport properties

\begin{tabular}{lccccc}
\hline \multicolumn{1}{c}{ Gas } & $A_{S}, \mathrm{~Pa} \cdot \mathrm{s} / \mathrm{K}^{1 / 2}$ & $T_{S}, \mathrm{~K}$ & $R, \mathrm{~m}^{2} /\left(\mathrm{s}^{2} \mathrm{~K}\right)$ & $\gamma$ & $\mathrm{Pr}$ \\
\hline Argon & $1.93 \cdot 10^{-6}$ & 142 & 208.1 & 1.67 & 0.67 \\
Nitrogen & $1.41 \cdot 10^{-6}$ & 111 & 296.8 & 1.4 & 0.71 \\
\hline
\end{tabular}

\subsection{Flat-Plate Case Setup}

Nonequilibrium boundary conditions are applied on the surface of a flat plate for the flow variables $(T, \mathbf{u})$. The boundary condition for the pressure $p$ at the flat plate is zero normal gradient. At the inflow boundary, the freestream conditions are maintained throughout the computational process. At the top boundary and the outflow boundary, fluid is allowed to leave the computing region; this condition specifies that the normal gradients of the flow variables $(p, T, \mathbf{u})$ vanish at these boundaries. At the lower boundary in front of the flat-plate, a symmetry condition is applied to all flow variables. A schematic diagram of the boundary conditions applied in the flat-plate cases is shown in Fig. 1.

In the flat-plate simulations, the computational results are sensitive to the numerical mesh sizes near the leading edge. A typical mesh for a flat plate simulation is regular rectangular. A mesh independence analysis was conducted to find the final mesh for the convergence solution; the final mesh sizes are $\Delta x=0.1 \lambda_{\infty}$ and $\Delta y=0.1 \lambda_{\infty}$ for Becker's case [6], and $\Delta x=0.4 \lambda_{\infty}$ and $\Delta y=0.9 \lambda_{\infty}$ for Metcalf et al.'s case [7].

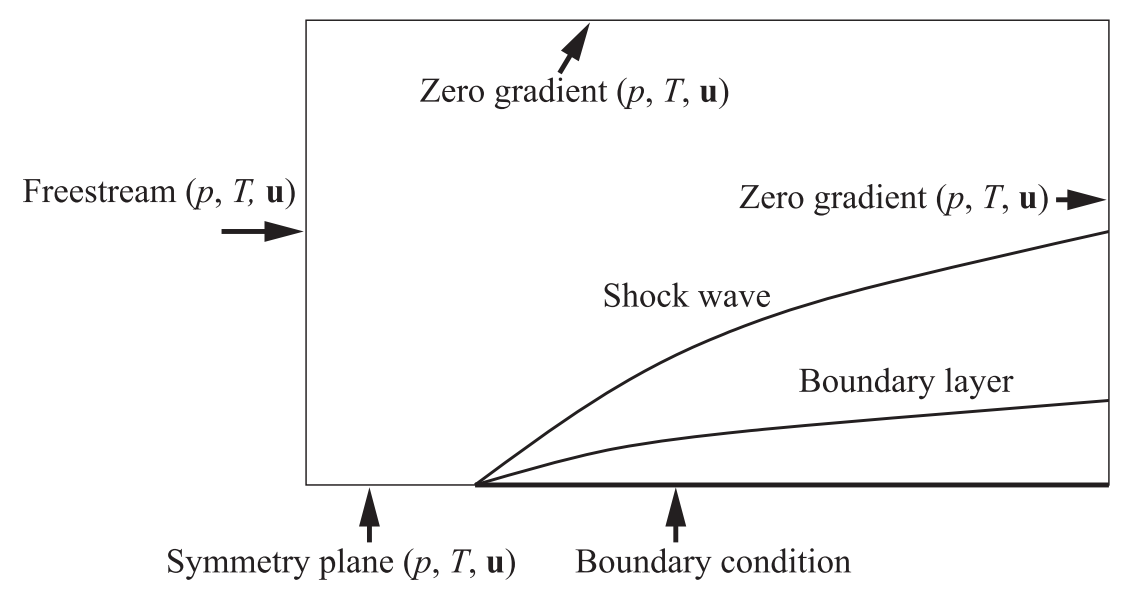

Figure 1 Numerical case setup for the flat plate problem 


\subsection{Circular Cylinder Case Setup}

A schematic diagram of the boundary conditions applied in the cylinder case is shown in Fig. $2 a$. The flow conditions for the circular cylinder DSMC case are as follows [5]: freestream conditions $\mathrm{Ma}=10, T_{\infty}=200 \mathrm{~K}, p_{\infty}=0.05 \mathrm{~Pa}$, the surface temperature $T_{w}=500 \mathrm{~K}$, diameter of cylinder $D=304.8 \mathrm{~mm}$, $\mathrm{Kn}=0.25$, and argon is the working gas. The computational mesh is built to wrap around the leading bow shock. The mesh is graded linearly over the first 100 cells near the surface, so that the mesh size varies from 10 to $100 \mu \mathrm{m}$. The smallest cell size near the surface is $\Delta x=10 \mu \mathrm{m}, \Delta y=2.4 \mathrm{~mm}$ which is approximately the same as the smallest cell size in [5]. A typical mesh of cells for the cylinder simulations is shown in Fig. $2 b$.

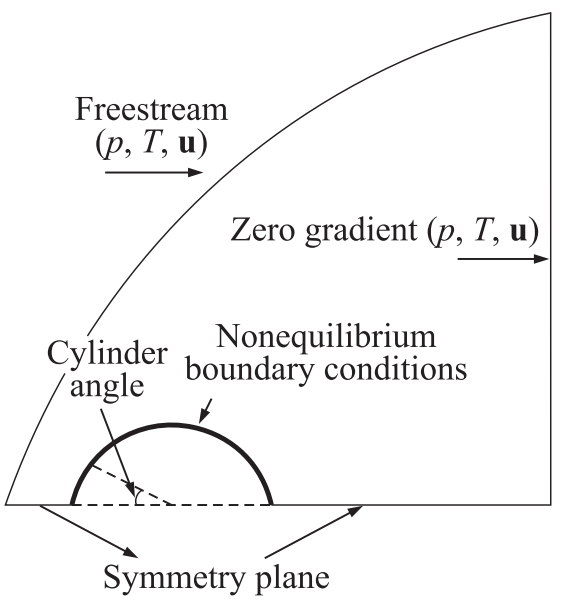

(a)

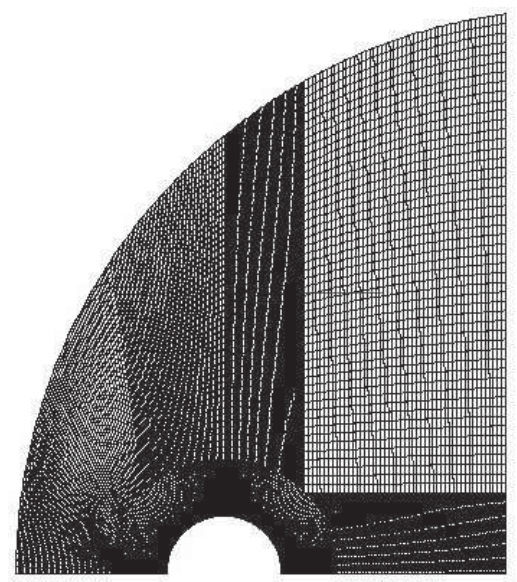

(b)

Figure 2 Setup of circular cylinder case: (a) numerical case setup for the cylinder in cross-flow; and (b) computational mesh for the cylinder case with 40,000 cells

\section{RESULTS}

\subsection{Flat-Plate Experimental Cases}

For the flat-plate case, three simulations were carried out as follows: first using the M/S boundary conditions; second using second-order slip velocity and the Smoluchowski temperature jump condition with $\sigma_{T}=1$ (chosen from the results obtained from the first case); and third with no-slip/jump conditions. Different values of the coefficients $\sigma_{u}, \sigma_{T}$ varying from 0.7 to 1 in the M/S boundary 
conditions, and of $A_{1}, A_{2}$ in the second-order velocity slip boundary condition, were employed to attempt to find the best values. Here, the results for surface pressure in the Metcalf et al. case [7] are presented vs. $x / \lambda_{\infty}=\mathrm{Kn}^{-1}$ as an illustration.

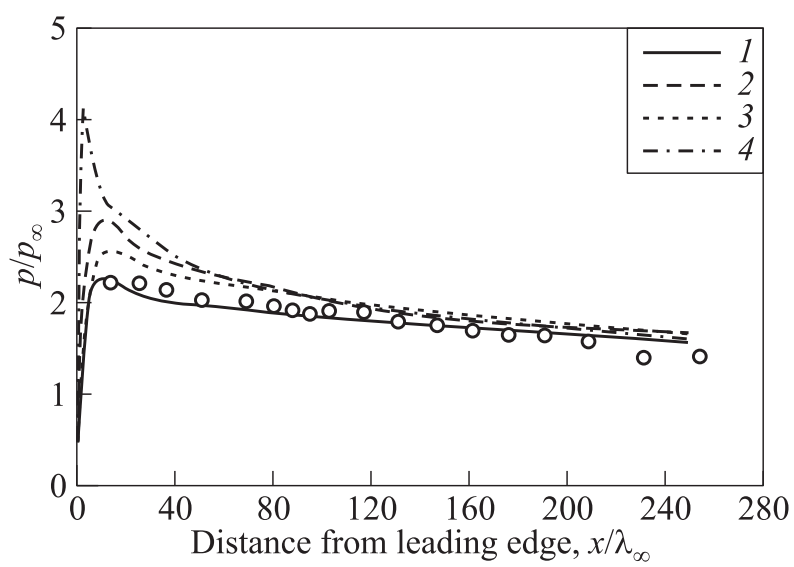

(a)

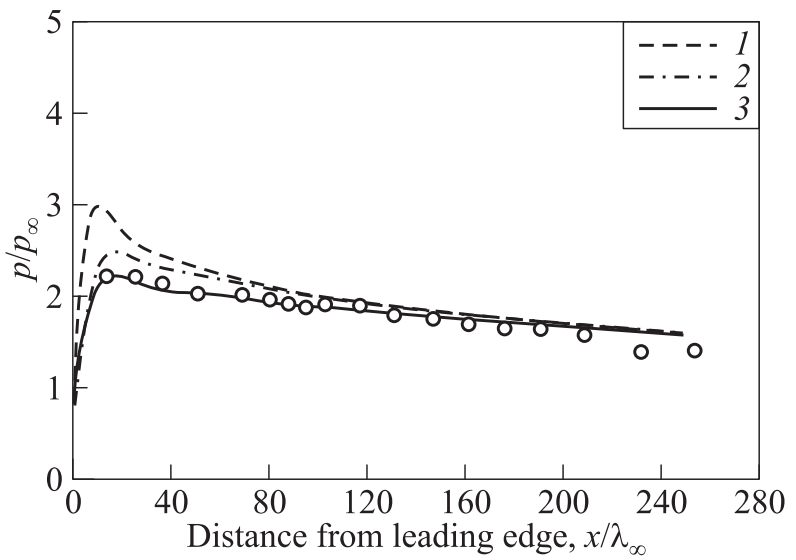

(b)

Figure 3 Metcalf et al.'s case [7], surface pressure distributions along the flat plate, simulations with various boundary conditions: $(a)$ using the M/S boundary conditions $\left(1-\sigma_{u}=0.7\right.$ and $\sigma_{T}=1 ; 2-\sigma_{u}=\sigma_{T}=0.8 ; 3-\sigma_{u}=\sigma_{T}=0.7$; and $4-$ no-slip, no temperature jump), and (b) using the second-order slip condition with the Smoluchowski temperature jump condition, $\sigma_{T}=1\left(1-A_{1}=1\right.$ and $A_{2}=0.5 ; 2-$ $A_{1}=1$ and $A_{2}=1.5 ;$ and $3-A_{1}=1.5$ and $\left.A_{2}=1\right)$. Symbols refer to experimental data 


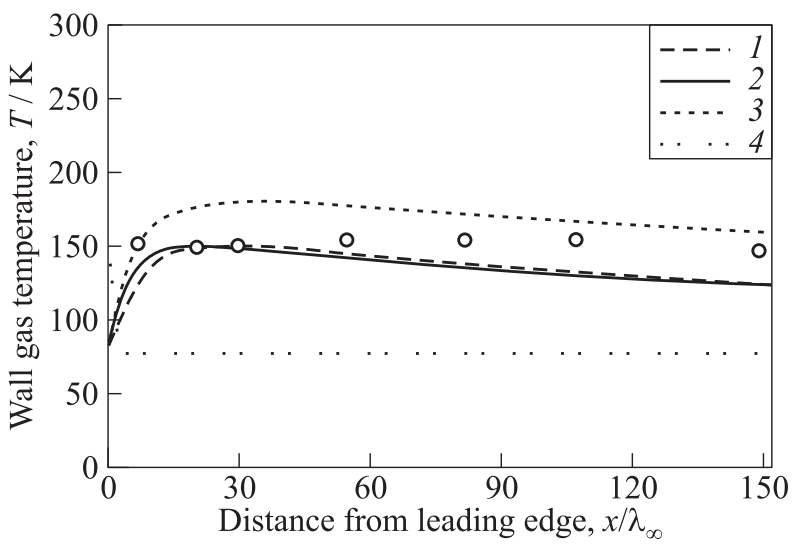

Figure 4 Metcalf et al.'s case [7], surface temperature distribution along the flat plate, simulations with various boundary conditions: $1-A_{1}=1.5, A_{2}=1$, and $\sigma_{T}=1 ; 2-\sigma_{u}=0.7$ and $\sigma_{T}=1 ; 3-\sigma_{u}=\sigma_{T}=0.7$; and $4-$ no-slip, no temperature jump. Symbols refer to experimental data

For the M/S boundary conditions, simulations are run with $\sigma_{u}=\sigma_{T}=0.7$ and 0.8 , and also $\sigma_{u}=0.7$ and $\sigma_{T}=1.0$. The combination of values $\sigma_{u}=0.7$ and $\sigma_{T}=1$ give the best agreement with the experimental data for $x / \lambda_{\infty} \geq 15$ $(\mathrm{Kn} \leq 0.067)$. There is a large difference between no-slip/jump results and the experimental data at $x / \lambda_{\infty} \leq 40(\mathrm{Kn} \geq 0.025)$, as seen in Fig. $3 a$. For the second-order velocity slip boundary condition, simulations are done with different values of $A_{1}$ and $A_{2}$, as seen in Fig. $3 b$. The values $A_{1}=1.5$ and $A_{2}=1$ give the best agreement with the experimental data of the surface pressure. The second-order term affects the surface pressure, in that increasing the value of $A_{2}$ decreases the pressure. For the surface temperature, the simulations with the $\mathrm{M} / \mathrm{S}$ boundary conditions with $\sigma_{u}=0.7$ and $\sigma_{T}=1$, and the second-order slip condition with $A_{1}=1.5, A_{2}=1$, and the Smoluchowski boundary condition with $\sigma_{T}=1$ give good predictions at the leading edge. The value $\sigma_{T}=1$ is better than the value $\sigma_{T}=0.7$ in predicting the surface temperature at the leading edge. The no-slip/jump results give the surface temperature $77 \mathrm{~K}$ along the flat plate, as seen in Fig. 4.

In the Becker case [6], the values $\sigma_{u}=0.7$ and $\sigma_{T}=1$ in the M/S boundary conditions and the values $A_{1}=1.5$ and $A_{2}=1$ in the second-order velocity slip boundary condition give the best agreement with the experimental data of the surface pressure for $x / \lambda_{\infty} \geq 18(\mathrm{Kn} \leq 0.055)$. There is a large difference between the no-slip/jump results with experimental data at $x / \lambda_{\infty} \leq 50(\mathrm{Kn} \geq 0.02)$, as seen in Fig. $5 a$. For the slip velocity, the values $\sigma_{u}=\sigma_{T}=0.8$ in the M/S boundary condition give the best agreement with the experimental data, as seen 


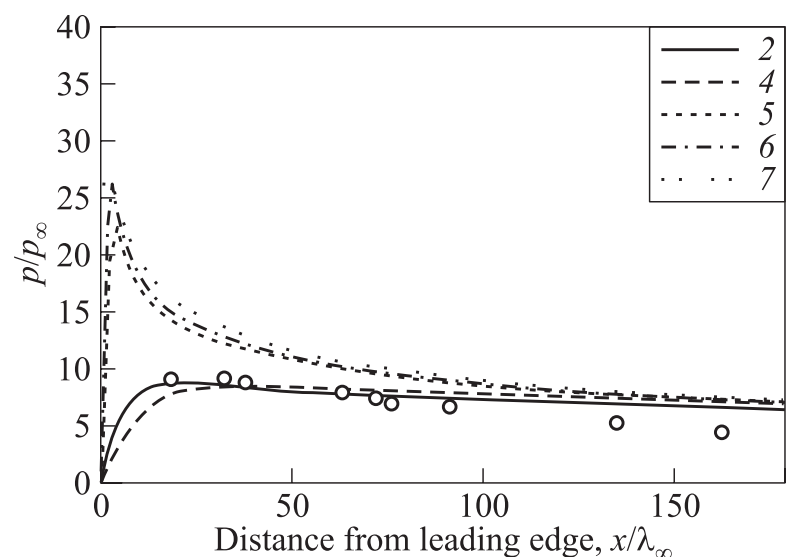

(a)

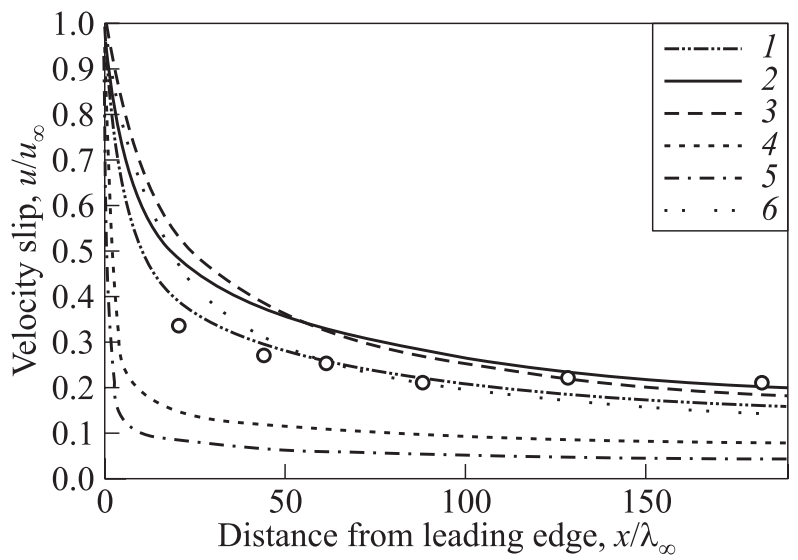

(b)

Figure 5 Simulation results of Becker's flat-plate case [7]: (a) surface pressure distribution along the flat plate, simulations with various boundary conditions; and (b) slip velocity along the flat plate, simulations with various conditions: $1-\sigma_{u}=\sigma_{T}=0.8$; $2-\sigma_{u}=0.7$ and $\sigma_{T}=1 ; 3-A_{1}=1.2, A_{2}=1$, and $\sigma_{T}=1 ; 4-A_{1}=1.5$, $A_{2}=1$, and $\sigma_{T}=1 ; 5-$ Langmuir boundary conditions, Covalent $d=0.21 \mathrm{~nm} ; 6-$ Langmuir boundary conditions, Van der Waals $d=0.294 \mathrm{~mm}$; and 7 no-slip, no temperature jump. Symbols refer to experimental data

in Fig. $5 b$. For the second-order slip condition, the values $A_{1}=1.2$ and $A_{2}=1$ give good agreement with the experimental data. In this case, the Langmuir boundary condition has also been applied, using, in turn, both the "Covalent" and the "Van der Waals" diameters $d$ of the gas molecule. The Covalent diameter is a measure of the size of the molecule which forms part of a covalent bond, 
and the Van der Waals is the diameter of an imaginary equivalent hard sphere molecule. The authors' initial results show that the Langmuir model is sensitive to the mean area of a surface site, $A=N_{A} \pi d^{2} / 4$ [3], and the heat of adsorption $D_{e}$. The results do not give as good agreement with the experimental data as the $\mathrm{M} / \mathrm{S}$ boundary conditions. More investigations into the appropriate values for $A$ and $D_{e}$ from the experimental literature may be needed to yield better and more reliable results.

\subsection{Circular Cylinder Direct Simulation Monte Carlo Case, $\mathrm{Kn}=0.25$}

In the circular cylinder case, the coefficient values $\sigma_{u}=0.7$ and $\sigma_{T}=1$ were tested in the $\mathrm{M} / \mathrm{S}$ conditions, obtained from the flat-plate case, with and without curvature effect [13], i. e., without including the $\boldsymbol{\Pi}_{m c}$ term in Eq. (5), and compare with the no-slip/jump results and DSMC data [5].

For the surface pressure, the values $\sigma_{u}=0.7$ and $\sigma_{T}=1$ give good agreement with the DSMC data at the stagnation point $\left(\varphi=0^{\circ}\right)$ while the no-slip/jump case does not (Fig. 6a). The curvature effect does not strongly affect the surface pressure. There is a difference between DSMC data and the present CFD results of simulations with the $\mathrm{M} / \mathrm{S}$ conditions at $30^{\circ} \leq \varphi \leq 180^{\circ}$, while the no-slip/jump results agree with DSMC data in this region. For the slip-velocity, simulations with and without curvature effect disagree with the DSMC data at $\mathrm{Kn}=0.25$. The results show that the curvature strongly affects the slip velocity at $\varphi \geq 60^{\circ}$, acting to reduce the peak value of the slip velocity (Fig. 6b).

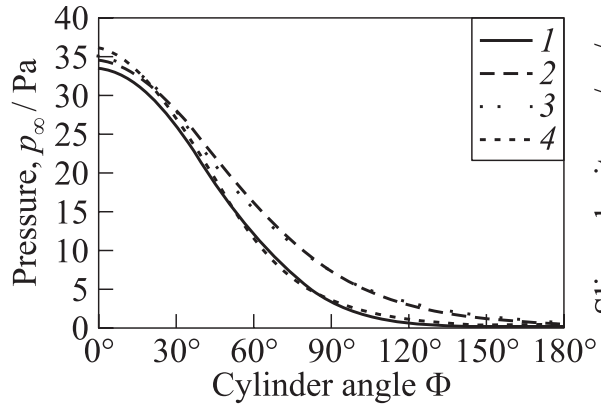

(a)

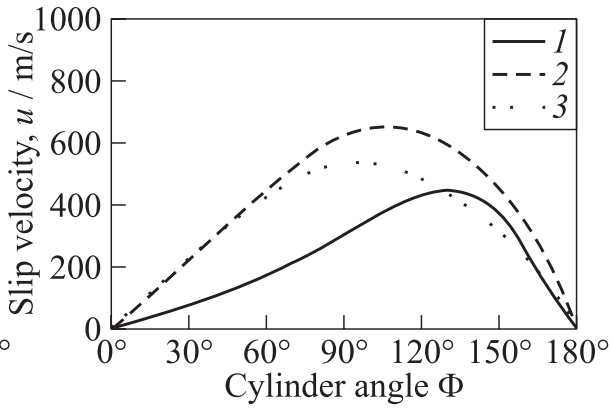

(b)

Figure 6 Simulation results of the circular cylinder in cross-flow case: $(a)$ surface pressure distribution, and (b) slip velocity: $1-\mathrm{DSMC} ; 2$ and $3-\sigma_{u}=0.7, \sigma_{T}=1$, without and with curvature, respectively; and 4 - no-slip, no-jump 


\section{CONCLUDING REMARKS}

The present results show that including velocity slip/temperature jump boundary conditions can improve CFD prediction of high-speed rarefied flows. The values $\sigma_{u}=0.7, \sigma_{T}=1$ in the $\mathrm{M} / \mathrm{S}$ boundary conditions, and $A_{1}=1.5$ and $A_{2}=1.0$ in the second-order slip condition with $\sigma_{T}=1.0$, give best agreement with the experimental data of the surface pressure up to $\mathrm{Kn}=0.1$ for the flatplate cases. The values $\sigma_{u}=0.7$ and $\sigma_{T}=1$ also give good agreement with DSMC data of the surface pressure at the stagnation point in the cylinder case.

The Langmuir boundary condition overpredicts the surface pressure and underpredicts the slip velocity. But the results are sensitive to the values of $A$ and $D_{e}$, and more appropriate values from experimental literature may improve these results.

From the results obtained in the flat plate case, the N-S-F equations seem to work well with the $\mathrm{M} / \mathrm{S}$ boundary conditions, or the second-order velocity slip and the Smoluchowski boundary conditions, up to $\mathrm{Kn}=0.1$. The secondorder velocity slip boundary condition is not much better than the conventional Maxwell slip boundary condition with the N-S-F model.

The circular cylinder case shows that the $\mathrm{N}-\mathrm{S}-\mathrm{F}$ equations with the M/S boundary conditions did not predict well the slip velocity at $\mathrm{Kn}=0.25$, as compared to DSMC data. This case shows that the curvature effect is important when simulating the slip velocity in blunt geometries.

\section{ACKNOWLEDGMENTS}

Nam T. P. Le would like to thank Dr. R. S. Myong and Prof. B. C. Eu for helpful discussions, and the James Weir Foundation and the University of Strathclyde for research scholarships that support this work.

\section{REFERENCES}

1. Maxwell, J. C. 1879. On stresses in rarefied gases arising from inequalities of temperature. Phil. Trans. R. Soc. Part 1. 170:231-56.

2. Von Smoluchowski, M. 1898. Über wärmeleitung in verdünnten gasen. Annalen der Physik und Chemie 64:101-30.

3. Myong, R.S. 2004. Gaseous slip models based on the Langmuir adsorption isotherm. Phys. Fluids 16(1):104-17.

4. Hadjiconstantinou, N. G. 2003. Comment on Cercignani's second order slip coefficient. Phys. Fluids 15(8):2352-54. 
5. Lofthouse, A. J. 2008. Nonequilibrium hypersonic aerothermodynamics using the direct simulation Monte Carlo and Navier-Stokes models. Ph.D. Thesis. University of Michigan.

6. Becker, M. 1969. Flat plate flow files and surface measurements from merged layer into transition regime. 7th Symposium (International) on Rarefied Gas Dynamics Proceedings. 515-28.

7. Metcalf, D. S., C. C. Lillicrap, and C. J. Berry. 1969. A study of the effect of surface temperature on the shock-layer development over sharp-edge shapes in lowReynolds-number high-speed flow. 7th Symposium (International) on Rarefied Gas Dynamics Proceedings. 619-34.

8. Weast, R. C. 1984. CRC handbook of chemistry and physics. Florida, USA: CRC Press Inc.

9. 2009. www.opencfd.co.uk/openfoam/.

10. Greenshields, C. J., H. G. Weller, L. Gasparini, and J. M. Reese. 2010. Nonoscillatory central schemes for high speed viscous flows. Int. J. Numer. Meth. Fluids 23:1-21.

11. Lilley, C.R., and M.N. Macrossan. 2002. DSMC calculations of shock structure with various viscosity laws. 23rd Symposium (International) on Rarefied Gas Dynamics Proceedings. 663-70.

12. 2008. http://www.imnoeng.com/flow/gasviscosity.htm.

13. Lockerby, D. A., J. M. Reese, D. R. Emerson, and R. W. Barber. 2004. Velocity boundary condition at solid walls in rarefied gas calculations. Phys. Review E 70:017303. 